\title{
El devenir del ciberespacio como medio de comunicación e interacción, una revisión documental a través de los usos comunicacionales de la plataforma sociodigital Facebook como medio social
} The evolution of cyberspace as a means of communication and interaction,
documentary review through the communicative uses of the digital social
platform Facebook as a social medium

Francisco Xavier Sánchez Hernández*

Investigador independiente

José María Rico núm. 128, Col. Actipan, Benito Juárez, C. P. 03230,

Ciudad de México, México

\section{Editor: Rogelio del Prado Flores}

Fecha de recepción: 17 de septiembre de 2021

Fecha de aceptación: 30 de noviembre de 2021 xaviery@gmail.com

https://orcid.org/0000-0002-1872-0677

\section{RESUMEN}

El ciberespacio se encuentra en expansión tanto por las actividades sociodigitales que ahí ocurren, como por los usos que las personas, instituciones y empresas le dan. Compuesto de una estructura digital ilimitada, ofrece diversos servicios para diferentes áreas del quehacer humano. Una de estas formas de interacción son las redes sociodigitales, que se erigen como parte de los medios digitales sociales. El objetivo es revisar, contrastar y destacar hallazgos de diferentes estudios respecto a los usos comunicacionales de Facebook, atendiendo a los principales enfoques para analizar el fenómeno. La metodología realizada fue una revisión documental de artículos de investigación académica en diferentes plataformas científicas y revistas indexadas buscando las características, las temáticas y usos que se dan a Facebook como medio de comunicación. Los resultados presentan una utilización heterogénea de la plataforma, sin embargo, aunque se observa un fuerte uso

\footnotetext{
* Maestro en Comunicación y Educación por la Universidad Autónoma de Barcelona (UAB). Cursa el Doctorado en Investigación de la Comunicación en la Universidad Anáhuac México, Campus Norte. Ha sido catedrático para diferentes universidades en el área de comunicación y medios. Fundador y director de empresas en comunicación y tecnologías. Director en áreas de medios y comunicación en la iniciativa privada y en instituciones públicas. Se ha desempeñado como productor digital y consultor en tecnologías de la información para diferentes empresas. Ha sido Ted Talker y ha participado en congresos y ponencias sobre temas de comunicación y medios digitales.
} 
del medio aún faltan por construir estrategias adecuadas de comunicación y aprovechar mejor el recurso a nivel interactivo.

Palabras clave: comunicación, ciberespacio, medio de comunicación, red sociodigital, medios digitales.

\section{ABSTRACT}

Cyberspace is expanding both because of the sociodigital activities that occur there, as well as the uses that people, institutions and companies give to it. Composed of an unlimited digital structure, it offers diverse services for different areas of human endeavor. One of these forms of interaction are sociodigital networks, which are established as part of social digital media. The objective is to review, contrast and highlight findings from different studies regarding the communicational uses of Facebook, taking into account the main approaches to analyze the phenomenon. The methodology carried out was a documentary review of academic research articles in different scientific platforms and indexed journals looking for the characteristics, themes and uses of Facebook as media. The results show a heterogeneous use of the platform, however, although a strong use of the medium is observed, there is still a need to build appropriate communication strategies and take better advantage of the resource at the interactive level.

Keywords: communication, cyberspace, media, socio-digital network, digital media.

\section{INTRODUCCIÓN}

Las tecnologías de la comunicación e información contribuyen a lo cultural y a la sociedad en gran medida porque proponen y facilitan principalmente la comunicación entre las personas (Caron y Caronia, 2007). Así la comunicación, una actividad que supone el intento de compartir y de relacionarse con los demás individuos en una sociedad (Wolton, 2006) se convierte en una actividad social plenamente identificada que involucra la producción, trasmisión y recepción de formas simbólicas (Thompson, 1998).

Las nuevas tecnologías han traído novedosas maneras de posibilitar la comunicación entre las personas, a través de diferentes procesos que involucran y evidencian el intercambio, producción y consumo simbólico, que se caracteriza por un nuevo ambiente tecnológico, los sujetos que participan e interactúan en él y la forma reticular que presentan las diferentes tecnologías digitales (Scolari, 2008). En este entramado digital tienen lugar diferentes actividades sociales y técnicas de las personas, así que ha tomado distintas formas 
de presentación, usos, reproducción y almacenamiento de conocimiento e información a nivel simbólico como menciona Sccolari, pero también a nivel cultural e incluso comercial en un amplio margen económico de la vida cotidiana. Así las plataformas sociodigitales no solo plantean valores simbólicos para los usuarios sino también económicos. Sobre esto Van Djick (2OI2) menciona que los valores monetarios que generan las plataformas sociales también pueden entenderse como una economía de lo social. Lo que estaría relacionado a diferentes factores que ocurren dentro de la plataforma, entre otros, la capacidad de monetizar las conexiones, es decir, entender la lógica entre las conexiones y la ingeniería de conexiones Van Djick (20I2).

Este espacio se ha constituido gracias a la tecnología del internet, y se ha definido como el ciberespacio, que se presenta como un espacio en red (Lévy, 2007), en esta esfera de lo digital toman fuerza nuevas configuraciones sociales, culturales, económicas y políticas.

Es así que la tecnología y sus procesos de evolución constantes y continuos han logrado transformaciones en diferentes ámbitos sociales y culturales. Por ejemplo, los medios de comunicación que sufrieron transformaciones al grado de constituirse en diferentes hibridaciones (McLuhan, 1996), que despiertan a su vez nuevas fronteras de producción e interacción en la forma de comunicarse, tanto como enviar y recibir mensajes o contenidos de diversas propiedades y características entre los usuarios. De esta forma un mismo contenido o mensaje puede propagarse o difundirse a través de las nuevas tecnologías mediáticas por canales muy diversos y reconstruirse bajo configuraciones muy distintas al llegar a la zona de recepción (Jenkings, 2008).

El ciberespacio se ha consolidado como un nuevo medio de comunicación producto de la creciente emergencia de la interconexión informática global que ejercen las personas entre sí (Lévy, 2007). Y es a través de las comunidades virtuales que se dan lugar en el ciberespacio, que este reformula la forma de comunicarse entre los sujetos. Una de estas nuevas formas que reorganizan la comunicación en la sociedad en este formato reticular hiperconectado son las llamadas redes sociodigitales, capaces de ofrecer interconectividad digital a las personas que se inscriben a ellas, fomentando en principio la comunicación y las comunidades de sujetos a través de la conectividad digital.

Facebook ha sido un medio social en constante cambio, con frecuentes innovaciones y que se ha mantenido de forma hegemónica en el ciberespacio; es así que los cambios y sucesos que se presentan en Facebook suelen ser de alta relevancia dada su preponderancia y constituyen un modelo a seguir imponiendo estructuras, modas y dando sentido a las prácticas de interacción virtual.

Facebook ha sido uno de los principales precursores en el ciberespacio como un medio social capaz de la reunión de diferentes grupos sociales a nivel global. 
Por esto se ha seleccionado la red sociodigital Facebook, dada la preponderancia actual como medio social, por su estructura y tipología que como herramienta social facilita la interacción e integración de diversas comunidades, además de mantener un predominio sobre las diferentes redes sociodigitales en la actualidad.

Este trabajo tiene un abordaje teórico contemplando las redes sociodigitales como un proceso cibercultural que se gesta en el ciberespacio producto de la realidad tecnológica actual de internet, la digitalización y la virtualidad. Desde una perspectiva teórica que contrasta con el viejo modelo comunicacional de la comunicación de masas, donde los mensajes fluyen de uno a muchos para pasar al que plantean las redes sociodigitales donde la comunicación es de muchos a muchos, uno a muchos o muchos a uno. Una configuración o estilos de comunicación más interesante que derrumba el clásico paradigma del broadcasting, uno a muchos (Scolari, 2028).

Un profesional de la comunicación debe estar en el entendido de la dimensión de los cambios e innovaciones que las TIC inyectan a la sociedad, debería cuestionar cuál es el nivel de importancia y la proporción en que las tecnológicas trasfiguran las formas de la conversación y las relaciones personales. De igual manera, conocer los vehículos que transportan los mensajes y qué ocurre con la materia de esos mensajes. Esto incluso entendiendo los diferentes contextos no solo tecnológicos, sino culturales, sociales, económicos e históricos. Un ejemplo de ello son los cambios y alteraciones sociales provocados por la pandemia de la Covid-ig.

Una vez entendidas y reformuladas las preguntas necesarias sobre las diferentes transformaciones mediáticas posibles, el profesional e investigador de la comunicación podrá entender de mejor manera el impacto de estos usos sociales que derivan del ciberespacio. Como menciona Del Prado (2018), la comunicación tiene que ver con la reunión de las personas en comunidad y en ese sentido el comunicar habla de una penetración de lo social.

\section{OBJETIVO}

Este artículo tiene como objetivo definir y catalogar qué usos le está dando la sociedad al ciberespacio en términos mediáticos, es decir, como un medio de comunicación e información. Esto planteado desde el enfoque que suponen las plataformas sociodigitales, en particular la red sociodigital Facebook y su impacto en la sociedad como un medio social comunicacional.

En síntesis, en esta revisión documental se propone encontrar e indagar cómo es que se está empleando la red sociodigital dentro del ciberespacio como medio de comunicación, y determinar para qué temas, intereses o actividades mediáticas, la página web de Facebook 
está sirviendo a los usuarios y comunidad en general. Por lo tanto, con este trabajo se busca examinar la situación actual de esta plataforma sociodigital y plantear una discusión y reflexión frente a posibles y futuras trasformaciones que el ciberespacio ejerce sobre usuarios, formatos, aplicaciones y demás servicios o productos digitales. Esto en función de la evolución o cambios en correlación a diferentes dimensiones, como la económica, la cultural, la social o la política, de las cuales el ciberespacio suele participar.

En este sentido, este trabajo busca abonar a la presentación del estado actual del medio sentando antecedentes para posteriormente observar en contraste los posibles cambios o evoluciones, en particular con las plataformas sociodigitales desde una perspectiva histórica-contextual así como operativa del medio, que permita entender características puntuales de esos cambios o evoluciones. Así, servir para diferentes propósitos, pero sobre todo para el investigador y profesional de la comunicación, pues las constantes mutaciones de los dispositivos, software, aplicaciones, apropiaciones y usos del ciberespacio son igualmente un reto. Por último y como elemento fundamental para este trabajo, es aportar al análisis de usos, apropiaciones y actividad que instituciones, empresas y organizaciones en general dan a la plataforma, para que los profesionales en comunicación puedan establecer parámetros de estos cambios o evoluciones en una relación y convergencia conceptual que aborde las características comunicacionales utilizadas al momento de esta investigación y así poder aproximar de mejor manera los alcances de la comunicación dentro de un medio social como lo es Facebook.

\section{MARCO TEÓRICO CONTEXTUAL}

\section{Los nuevos medios de comunicación}

Los medios de comunicación masiva en virtud de los aportes tecnológicos del momento superaron el tipo de comunicación donde era indispensable la interacción de forma presencial, sin embargo, debido a esto, es muy difícil determinar los receptores que son participes del acto comunicativo (Luhmann, 2000). Los receptores de los mensajes se encuentran diseminados, indistinguibles y son considerados capaces de un mínimo de habilidad de decodificación frente a mensajes que se plantean básicos y con un común denominador pues son emitidos para una masa heterogénea (Lévy, 2007).

Los nuevos medios de comunicación han sido posibles debido a la emergente evolución de la tecnología de internet y la digitalidad. Este nuevo contexto mediático llama al interés del pensamiento social no solo en términos de una cultura global, sino en términos de nuevos formatos de expresión y relación social. 
Por medios de comunicación se comprende lo referente al soporte o lo que transporta el mensaje, el vehículo (Lévy, 2007). En este caso el ciberespacio es una nueva forma de vehiculizar los mensajes de las personas.

\section{El ciberespacio como lugar de reunión e interacción}

Cuando Internet, es decir, la World Wide Web fue inventada, Berns Lee la fundó abierta y universal, sin ningún tipo de restricción o división, lo que significó para su momento un espacio accesible desde cualquier lugar sin ningún tipo de muro que imposibilitara su entrada (Rovira, 2017). Es decir que el ciberespacio se desarrolló y propagó de una forma sencilla y rápida gracias a esta tecnología de la red de redes como también es conocida la internet. Es en ese sentido que el ciberespacio se entiende no solo por la infraestructura técnica y material, sino también por el basto universo de informaciones que ahí habitan, los actores que lo componen al navegar por él y que son quienes lo sustentan y alimentan (Lévy, 2007).

De esta forma el ciberespacio se aprecia como un espacio público y de acción, dado que los sujetos pueden interactuar en él (Asencio y Navío, 20 I7), lo que conlleva a pensar en las implicaciones culturales que emergen de esta interacción social virtual, esto desde las prácticas y las experiencias sociales que se pueden recrear. Entender que el ciberespacio surge como desplazamiento producto de un medio atemporal y sin fricción (Piscitelli, 2002) y que ostenta características muy propias y particulares. Incluso al paso del tiempo es posible inferir que estos desplazamientos hacia lo digital fomenten una mutación o transformación en la conducta de las personas, cambios que pueden ocurrir en distintas dimensiones, como la económica, sociológicas o psicológicas por mencionar algunas (Bernete y Cadilla, 2019).

En cuanto a su acceso y tecnologías implicadas, existe un proceso de diversificación y simplificación de las interfaces que, sumado a las bondades de la digitalización de las cosas, el ciberespacio es capaz de extender y reproducir los accesos a su entorno para diversos actores (Lévy, 2007). Esto permite al ciberespacio convocar nuevas realidades al integrar los medios de comunicación cultural previos y también integrar nuevas características como lo es fortalecer el volumen de memoria, imaginación, razonamiento, percepción y creación (Tubella y Vilaseca, 20I3) que permiten establecer otro tipo de relaciones interpersonales, de trabajo, diversión, entretenimiento, etc. Por tanto, las tecnologías que posibilitan estas acciones no son exclusivamente artefactos, sino todo un nuevo implemento que se inventó como parte de una nueva práctica (Piscitelli, 2002).

Bajo estas condiciones es posible precisar al ciberespacio como un lugar o mecanismo con una fuerte capacidad socializadora y aglutinadora que como resultado permite analizar 
y examinarlo como una plataforma de generación de espacio que es de todos, es decir, un espacio compartido (Asencio y Navío, 2017).

Lo que lleva a pensar en el ciberespacio como un conjunto de acciones, experiencias que irremediablemente predisponen a pensar en actividades culturales, con nuevas extensiones, donde incluso las formas de participación actuales también se ven transformadas para establecerse como ciberculturas (Tubellay Vilaseca, 20I3). En la apropiación del ciberespacio que las ciberculturas toman forma para entender las dinámicas sociales que los sujetos enfrentan, de las que son participes y de las que son artífices. Además con el tiempo y las diferentes interacciones que se suscitan en su ambiente, el ciberespacio con sus diferentes movimientos ciberculturales muestra que no solo está constituido por información, materiales y seres humanos, sino también constituido de programas (Lévy, 2007), provocando que sea un espacio que en su transformación y alteración se preste de incluir o funcionar con la implantación de normas y a su vez vaya hacia la codificación del mismo (Asencio y Navio, 2017).

De esta forma, como menciona Rovira (2017), en el crecimiento y desarrollo de la red, el ciberespacio comenzó a tener cercados, o sea, una forma de crear confines o divisiones y estructuras que dan orden pero que también imponen reglas, incluso para acceder al mismo. Lo que permitió entre otras cosas que florecieran las relaciones de todo tipo hasta llegar a las relaciones cliente-servidor, donde una entidad situada en el ciberespacio propone un espacio pero a su vez puede administrar servicios, servidores e incluso los contenidos (Kleiner, 20II). De una forma categórica y axiomática, el ciberespacio se presenta con una arquitectura de la participación, misma que está implícita en su esencia y que es parte de la evolución de la misma red (O’Relly, 2005).

Lo que implica que el ciberespacio es fundamentalmente un encuentro para la negociación, la comunicación y la organización social, pero también se ha desplegado esa competencia de ejercer como un mercado para el acceso y transacción del conocimiento y la información (Lévy, 2007). En este sentido los servicios y el funcionamiento del sistema mejoran en el momento en que la gente concurre, se apropia y participa en las diferentes ciberculturas lo cual irremediablemente agrega valor (O’ Relly, 2005).

De forma integral y en sus propios términos, el ciberespacio es un mecanismo de comunicación, de interacción y de formación de comunidad que funciona como instrumento conveniente para la exposición y desenvolvimiento de la inteligencia colectiva (Lévy, 2007).

\section{Redes sociodigitales}

Dos de las principales potestades de las redes sociales son reconocer quiénes están conectados y con quiénes establecen esa conexión, es decir, identifica los vínculos que se crean entre 
los participantes de la red; y la otra es reconocer lo que circula a través de esas conexiones, es decir, lo que fluye entre esos vínculos (Christakis y Fowler, 2010). Esto en el entendido de que dentro de una red estas conexiones entre los usuarios se constituyen bajo demanda y tienen la particularidad de suspenderse a voluntad (Bauman, 20I2).

Las redes sociodigitales se presentan como una nueva forma de comunicación, una que se da de forma directa entre las personas y que identificando el proceso productivo que se genera en ellas, se debe considerar el contendido y el soporte tecnológico que en este caso es la propia red (Scolari, 2008), dado que este nuevo paradigma comunicacional tiene diferentes características que lo distinguen de cualquier otro medio. Las comunidades que se concentran en las redes sociodigitales pueden organizarse a la par de las mismas problemáticas o intereses, pero la geografía deja de ser un inconveniente para su creación (Lévy, 1999).

Otro cambio fundamental que proponen las redes sociodigitales es la presencia individual que la plataforma otorga al sujeto al permitirle tres elementos básicos: construir un perfil público o semipúblico dentro de un sistema delimitado, articular una lista de otros usuarios con los que comparten una conexión y ver y recorrer su lista de conexiones y las realizadas por otros dentro del sistema (Boyd y Ellison, 2007). Esto da independencia y con ello interés por la participación, pensado en una comunicación más directa y bidireccional, unos a unos y de unos a muchos o de muchos a muchos, configuraciones que la universalidad y la virtualización del ciberespacio le permiten a los usuarios. Y precisamente en ello es que las redes sociodigitales estimulan una economía del interés que queda en poder de los usuarios, el cual decide a quién seguir, a quién agregar, qué ver, qué compartir o qué publicar (Rovira, 2017).

\section{Internet y Facebook una plataforma social, convergente, mediática y disruptiva}

El ciberespacio ha planteado una serie de conversaciones que enfrentan la realidad de los medios digitales con las relaciones entre cultura, tecnología y sociedad (Scolari, 20I8). La virtualización ha permitido el desplazamiento de diferentes actividades sociales al entorno digital (Bernete y Cadilla, 2019), lo que irremediablemente hace voltear para identificar estas actividades, para entenderlas y anticipar las estrategias de comunicación necesarias para su manejo.

El ciberespacio ha manifestado una constante y permanente evolución en lo material y lo conceptual, uno de los conceptos más significativos y que es pieza fundamental del pensamiento de internet, es el que menciona Tim O’ Relly (2005), el espacio web en su versión 2.0 no presenta fronteras, sin embargo, sí presenta un núcleo gravitacional que incide en muchos de los aspectos de comportamiento frente a su uso. De tal forma que este núcleo 
lo presentan las grandes empresas que dan forma y concentran a grandes cantidades de usuarios. Con esto la arquitectura y el mapa del ciberespacio cambia para diferentes fines o intereses, el traslado o manejo de la información y recursos se va transformando. Ante las integraciones, materiales, conceptuales que se dan lugar en este espacio surgen las prácticas ciberculturales, manifiestos, confrontaciones, incertidumbres posmodernas y gestiones mercadológicas de lo que debe ser y ocurrir en el ciberespacio (Scolari, 20I8).

De acuerdo con el informe de estadísticas globales digitales realizado en sociedad entre las empresas Hootsuite y We are social (202I), los usuarios de internet a nivel global son 4.80 billones, con un crecimiento anual entre julio de 2020 y julio de $202 \mathrm{I}$ del $5.7 \%$, lo que significa 257 millones más de personas que se integran al uso de internet. Es decir, que el ciberespacio manifiesta un crecimiento constante y que integra a nivel global a cerca del $60 \%$ del total de la población mundial. Los internautas que dan uso, que navegan en internet, presentan diferentes motivaciones para estar conectados. Dentro de las cinco motivaciones más importantes que hay se encuentran: en primer lugar, la búsqueda de información, en segundo lugar, el mantenerse en contacto con amigos y familia, en tercer lugar, mantenerse actualizado respecto a noticias y eventos, en cuarto lugar, la búsqueda de cómo hacer cosas y por último ver videos, programas de televisión y películas (We are social \& Hootsuite, 202I).

Los sitios web así como las apps más usadas en el orbe son las redes sociodigitales. En relación con esto, dentro la lista de plataformas sociales más usadas y con mayor número de usuarios globales activos está Facebook, que ostenta el número uno de la calificación (We are social \& Hootsuite, 202I).

En el año 2004, Facebook nació como una plataforma que desplegaba páginas personales de los usuarios que se registraban en ella (solo presente en un entorno universitario) y que a su vez le permitía la creación de grupos. Para el año 2005, la red sociodigital permitía subir fotos a sus usuarios y solo un año después la plataforma se convirtió en una red pública y se integró a la red móvil a través de la conexión por medio de un teléfono celular (López y Ciuffoli, 20I2).

Esta rápida constitución y evolución en sus características permitieron a Facebook convertirse en la herramienta principal de comunicación masiva (Tapia, 20I6). Siguiendo en esta línea, la plataforma sigue en un constante cambio e innovación, recientemente la empresa ha lanzado un nuevo plan estratégico para la plataforma en general. Este plan reúne todas sus aplicaciones y tecnologías bajo una nueva marca de empresa llamada Meta, lo que denominan como el metaverso, que es su propuesta conceptual para la conexión social que busca ayudar a las personas a conectarse, encontrar comunidades y hacer crecer los negocios (Introducing Meta: A Social Technology Company, 202I). Es decir, Facebook, cambia para convertirse 
en Meta, una propuesta para el futuro y su consecuente desarrollo de tecnologías sociales que reúne tecnologías como la realidad virtual, ambientes $3 \mathrm{D}$, realidad aumentada e incluso hardware.

Esta evolución de la plataforma impacta en general al escenario sociodigital pues Meta otrora Facebook actualmente conforma un conglomerado de tecnologías y apps sociodigitales como lo son Whatsapp, Messenger, Instagram, Workplace, Novi, y aplicaciones que se presentan directamente en la plataforma sociodigital Facebook. Esto además de hacer la plataforma más plural y diversificada permite a los usuarios una mayor oferta de creación y usos. Como menciona López y Ciuffoli (2012) sobre Facebook, que es un espacio convergente que presenta la integración de diferentes formatos para poder ser publicados por diferentes tipos de usuarios que van desde el individuo en lo particular, celebridades, políticos, marcas, instituciones y organizaciones.

\section{Redes sociales y pandemia de la Covid-19}

A principios de 2020 se presentó la pandemia de la Covid-I9, sus características sanitarias sacudieron globalmente a las diferentes sociedades del orbe, provocando transformaciones de diversa índole, como fueron las de tipo comunicacional. Esto debido a la aplicación de una cuarentena que propició restricciones al contacto social y que limitó las relaciones de contacto físico-presencial, impactando los entornos laborales, de esparcimiento, familiares, etc. Esto dio un impulso a las tecnologías sociales, de comunicación e información, las cuales funcionaron como una opción de comunicación y conectividad a las personas, al menos a nivel virtual. Plataformas de redes sociodigitales manifestaron un crecimiento en su nivel de usuarios registrados. Así los usuarios activos de redes sociales crecieron en un I3.1\% en el periodo de julio de 2020 a julio de 202I, lo que significó un aumento de 520 millones de usuarios a nivel mundial, esto es, alrededor de casi 15.5 nuevos usuarios cada segundo (We are Social \& Hootsuite, 202I). Con esto se puede observar, al menos durante el espacio de confinamiento, que hubo un fuerte registro y aumento en las audiencias de diferentes redes sociales. Así, redes sociodigitales como Instagram en México crecieron vigorosamente, con un incremento del ${ }_{12} \%$ traducido en 3 millones de usuarios registrados (We are Social \& Hootsuite, 2020). De igual manera se registró un incremento en el uso de las redes sociodigitales, y el mayor incremento fue en dos grupos de usuarios divididos por edades, siendo el grupo de I6 a 24 años los de mayor incremento con $58 \%$ y para el grupo de edades entre 25 a 34 años con $44 \%$ (We are Social \& Hootsuite, 2020). Estas cifras permiten observar en este crecimiento un uso significativo de las redes sociodigitales sobre otros servicios dentro de las diferentes actividades en línea. 


\section{METODOLOGÍA}

El presente trabajo hace una indagación sobre los usos mediáticos de la plataforma sociodigital Facebook, es decir, la red sociodigital empleada como un medio de comunicación. Así de forma puntual, la información recabada procede de artículos donde se estudia e investiga a la red sociodigital como un medio social de comunicación. Así una primera etapa metodológica de este trabajo fue establecer este primer criterio de selección, que identifica y puntualiza a Facebook como un canal de comunicación, más allá de usos como el entretenimiento o medio de conectividad individual de los usuarios. Es decir, Facebook como medio institucional o empresarial de comunicación. A partir de esta primera selección se realizó una clasificación puntual para establecer las siguientes categorías en los estudios seleccionados: Uso de Facebook por temática o concepto, Uso de Facebook para comunicación política, Uso de Facebook como medio de comunicación de las organizaciones y Uso de Facebook como estrategia comunicativa. Así este trabajo de investigación se presenta bajo estas cuatro diferentes clasificaciones para delimitar y ordenar los resultados de forma más precisa y relacionados a su interpretación como un medio de comunicación una vez efectuada la documentación.

La realización de trabajo se hizo a través de la revisión documental en diferentes revistas científicas con una antigüedad máxima de cinco años. Los diferentes artículos fueron recuperados de diferentes bases de datos, estas fueron: ProQuest, Gale Onefile, Web of SScience Ebsco. Se realizó el registro y el estudio de estos trabajos para entregar un análisis de las formas en que la comunidad se apropia de Facebook para satisfacer sus necesidades de comunicación. Se aclara que para esta investigación se utilizaron solo artículos que expresamente utilizan la plataforma Facebook para su investigación, dado que dentro de la revisión documental también se encontraron otros trabajos pero que se enfocaban en otras redes sociales como Twitter o Instagram o simplemente los estudios hacían referencia al concepto general de redes sociodigitales. La selección de Facebook recayó en el tipo de plataforma que es, una red sociodigital horizontal, con un nivel preponderancia alto, facilidad de uso, la popularidad, su relevancia y características técnicas y sociodigitales. Además, conceptualmente y en la práctica constituye una plataforma completa y robusta que tiene alto nivel de tráfico web, agilidad para la constitución de comunidades online, acceso a servicios web para la economía social, mensajería instantánea y que ofrece un amplio margen de propósitos que van desde aspectos lúdicos hasta institucionales. 


\section{RESULTADOS}

La plataforma sociodigital Facebook tiene una gran recepción en el ciberespacio por diferentes entidades que van desde usos personales hasta corporativos. Sin embargo, se puede observar de acuerdo con esta revisión, que los investigadores pueden advertir que la plataforma no es usada en su máxima capacidad, en algunos casos el arribo a Facebook ha sido tardío, además de que faltan estrategias comunicacionales propias para desempeñar mejores prácticas. Sin embargo, uno de los usos más prominentes es el relacionado con la política, donde la plataforma sociodigital es muy usada para temas electorales, de campañas o de la conversación de políticos con la ciudadanía. Facebook ha abierto nuevas ideas para la investigación, como es pensar en nuevos planteamientos metodológicos, o para rescatar información y datos de los usuarios que participan en los grupos online. Muchas empresas encuentran un gran apoyo de la plataforma y los investigadores circunscriben muy bien las capacidades de Facebook y la correlacionan con problemáticas actuales y precisas.

Facebook se erige como una de las plataformas más usadas y presenta una gran diversificación de usos y apropiaciones que la comunidad sociodigital aprovecha para plantear la comunicación con una gran diversidad de temáticas, motivaciones e intereses. Este uso y apropiación de la plataforma va desde las empresas públicas y privadas para comunicarse con la sociedad.

El ciberespacio se revela como un complemento de una generación digital que no ve solo contenido, sino que usa los nuevos medios para participar en los movimientos sociales (Lago, Direito, Rodrígiuez y López, 20ı6).

La medición de las conversaciones a través de las interacciones logradas dentro de la plataforma son una metodológica altamente usada, así como el análisis del contenido de los mensajes que se realizan en los diferentes perfiles. La gran mayoría de los estudios tiene una tendencia por usar una metodología de análisis de contenido revisando las interacciones en los mensajes y publicaciones que se realizan bajo diferentes temáticas o disciplinas, lo que indica una homogenidad en las investigaciones.

Se puede advertir que la información que se desprende de opiniones y comentarios de las diferentes conversaciones de publicaciones y comunicación general de las empresas o instituciones por medio de Facebook es uno de los activos más importantes en cuanto a generación de información y datos que se pueden obtener de los propios usuarios.

Facebook se presenta como un espacio para investigar y analizar la comunicación digital de los medios sociales, en especial los que exponen información abierta como puede ofrecer la plataforma. 


\section{Usos por temática o conceptos}

Como espacio social, Facebook se abre a diferentes temáticas para satisfacer mediáticamente y de forma virtual la conversación y la acción social. Muchos de estos temas y conceptos son novedosos y en este apartado se integran. Actividad y presencia son conceptos permanentes en la gran mayoría de los estudios, fundamentalmente porque es una manera de medir o de establecer un análisis metodológico cuantitativo que aporte datos relevantes (Pastor y Paniagua, 2020; Losada y Capriotti, 2015; Amaral y Santos, 2020).

Otro estudio integra el concepto de bomofilia en conversaciones políticas que se celebran en la plataforma (Valera, Calvo y García, 2018). Se estudia el término de compromiso 2.0 a través de los flujos de información creados en Facebook para comprender la interacción y participación de ciudadanos con políticos (Rodríguez, Ruiz, Paíno, y Jiménez, 2017). También se estudian las implicaciones emocionales y actitudinales sobre el uso de la plataforma (Zumárraga, Valdivieso y Trujillo, 2020), además de hacer énfasis en la construcción de los imaginarios en la relación con las instituciones y actores políticos, presentando el concepto de outsider político para quienes hacen uso también de las TIC para temas políticos, en este caso con Facebook (Briceño, Manrique, Sanabria, y Gómez Reyes, 20I8). Facebook, a través de las diferentes herramientas que desarrolla para sus páginas personales y de empresa, ha abierto opciones que ayudan a, por ejemplo, predecir el comportamiento (David, Zhitomirsky-Geffet, Koppel, y Uzan, 20I6). Un concepto recurrente en las investigaciones revisadas es el de participación (Vromen, Loader, Xenos, y Bailo, 20I6; Gomes-Franco y Colussi, 2016). Losada y Capriotti (2015) comentan que el uso de las redes sociodigitales acerca a los museos al concepto de museo social o museo 2.0, mismo que concierne a un concepto más social y participativo de cara al público.

Los autores destacan la inmediatez y la capacidad de llegar a un público amplio y diferenciado respecto a la mediación cultural en este caso, contenido artístico, por medio de Facebook; además de usar la herramienta como mediadora también la refieren como un excelente medio de divulgación (Izquierdo, Álvarez, y Nuño, 20I7). Facebook permite abonar a los análisis de acontecimientos históricos a través de las interacciones que los usuarios hacen, los autores encontraron que se observan disputas y divisiones que ocurrieron en su momento el mundo offline pero que actualmente se han trasladado al ciberespacio (Pilgun y Dzyaloshinsky, 2017).

\section{Usos para comunicación política}

Un estudio cualitativo analiza el comportamiento de millennials que consumen información política en redes sociales y tv, esto a través del seguimiento de un grupo de Facebook que 
permitió entender la conducta de los jóvenes ante este tipo de información (Lago, Direito, Rodríguez, y López, 20I6); en este caso Facebook funciona como vehículo para entender el comportamiento frente a la comunicación. Un siguiente estudio analiza el uso de las redes sociodigitales con fines políticos, describiendo los clústeres de donde proviene la información así como el tipo de actor, analizando el comportamiento comunicativo y lingüístico de usuarios rusoparlantes (Pilgun y Gradoselskaya, 20I6). Otros estudios tratan de cómo Facebook es empleado para participar y promover la conversación online sobre temas políticos y sus implicaciones para la acción política (Valera et al., 20I8; Zumárraga et al., 2020). A su vez, se está estudiando la herramienta para analizar la interacción-participación entre actores políticos y sociedad, todo esto a través de las narrativas practicadas en Facebook (Rodríguez, Ruiz, Paíno, y Jiménez, 20I7). Sumado a esto, las relaciones con instituciones y actores se miden en el escenario democrático para entender la afección o desafección política (Briceño et al., 20I8). Con un análisis más instrumental de la comunicación política se examina la función y temática de los posts y la presencia e interacción midiéndola a través de recursos, bashtags y likes (Marcos, Viounnikoff-Benet, y Ripollés, 2020). Se ha declarado directamente que Facebook ha sido adoptado por los políticos para comunicar temas de campaña electoral (David et al., 20I6). En cuanto estudios directamente de políticos, existe un análisis longitudinal de la presencia de Barack Obama en Facebook. A través de su Fan Page los autores descubrieron que el político usó más el medio para temas políticos que para temas personales, sin embargo, tenía más respuesta a los artículos que tenían una carga emocional o de tono privado en cuanto a su persona que los de información política (Vučković y Črnič, 2020). Otro estudio mixto sobre el manejo de Facebook busca reconocer a los jóvenes que usan la plataforma para hacer política, esto es informarse, expresarse y tomar medidas, además investiga también el éxito del involucramiento de los políticos con los ciudadanos y su capacidad de movilización de jóvenes que participen sin conexión (Vromen et al., 20I6).

\section{Usos de Facebook en las organizaciones}

Uno de los estudios refiere que el uso y empleo de Facebook en 49 bibliotecas populares de la Plata, en Argentina, fue tardío. El año promedio de arribo a Facebook es el 2013, eso los acota a estar en una etapa de aprendizaje (Laudano, Corda, Planas, Kessler, y Aracri, 20r6). Otro estudio busca evaluar la presencia, actividad y escucha activa en Facebook de los museos de España y el mundo, a través de un instrumento que busca analizar el contenido de los post en estos tres aspectos mencionados (Losada et al., 2015). Para Arroyo, Calle y Van Wyk (20I8), las redes sociodigitales son la base de la eficacia de la comunicación de las ONDG, 
indican que Facebook es la red social más utilizada en campañas de emergencia. Su estudio mide el comportamiento del público a través de la interacción y un análisis de contenido de los mensajes sobre una campaña de un desastre natural. Concluyen que la eficacia de las campañas depende más de las interacciones a los mensajes que la cantidad de posts que se generen. Otro estudio habla de la comunicación de marcas de moda, marcas de lujo en una comparativa con las marcas de moda rápida; los investigadores analizan el contenido de las publicaciones y advierten que no se aprovecha las posibilidades de comunicación bidireccional que la plataforma ofrece (Navarro, García, y Segarra, 2020).

\section{Usos de Facebook como estrategia comunicativa}

Una de las principales características de Facebook es ser una fuente de información sobre diferentes áreas o disciplinas que aportan a estrategias en comunicación o a la investigación. En temas políticos Facebook está funcionando para la obtención de rasgos estadísticos que aportan los usuarios según el tipo de comunidad a la que se estudia generando datos para aumentar la efectividad de las campañas con anuncios dirigidos (David et al., 20I6). Un estudio presenta el análisis de la estrategia de comunicación de diferentes destinos turísticos a nivel mundial, a través de un análisis de contenido para de ahí examinar la actividad y la capacidad de generar comunidad e interacción. Resolvió que los sitios mantienen una presencia meramente instintiva y no una presencia con comunicación estratégica (Pastor y Paniagua, 2020). Por medio de una evaluación comparativa de estrategias comunicativas online que las universidades aplican en Facebook se realiza un análisis de contenido para obtener los diferentes niveles y gestión de la comunicación online, que implica relacionar factores como posición, el tamaño de la universidad, tráfico web y la antigüedad (Segura, Paniagua-Rojano y Fernández, 2020). Facebook se ha convertido en un excelente medio para la transmisión de cultura empleado como un divulgador y comunicador de contenidos artísticos (Izquierdo et al., 2017). Un estudio se enfoca en el empleo de Facebook en las universidades portuguesas como medio de comunicación institucional, el análisis que efectuaron permite ver se puede crear valor de marca en las universidades más pequeñas al usar la plataforma (Amaral et al., 2020). Otro estudio muestra como Facebook es usado como medio de comunicación alternativo para eventos, además se concentra en analizar las características de la divulgación del evento (Gomes-Franco et al., 2016). 


\section{CONCLUSIONES}

El ciberespacio presenta una oportunidad para la vertebración y la convergencia social (Asencio y Navio, 2017) y en ello existen implicaciones culturales que surgen de los usos y crecimiento constante de la tecnología. En este sentido las redes sociodigitales como herramientas de adquisición de información son altamente utilizadas por diferentes entidades. Funcionan como dispositivos o herramientas que almacenan, distribuyen y gestionan la información de la comunidad online.

El comunicador debe considerar en su trabajo integral como profesional de la comunicación el entorno mediático y atender a los diferentes mecanismos comunicacionales que se ofrecen actualmente para entender el impacto y problemáticas asociadas a esas apropiaciones y usos que se dan de los medios, entendiendo características como la universalidad del medio en contraste con la capacidad totalizadora del mismo. El ciberespacio como menciona $\mathrm{O}^{\prime} \mathrm{Re}$ lly (2005) es un espacio con una arquitectura libre y participativa en principio, pero que puede estar cercada (Rovira, 20I7) y sitiada de programas donde existen entidades que también hacen uso y se aprovechan de la inteligencia colectiva que ahí tiene lugar (Lévy, 2007; Pisani, 2006). Es decir, además de ser un medio social, las empresas han encontrado cómo visualizar un mercado social online que se favorece de la emergencia sociodigital de estar conectados.

El filósofo tunecino Pierre Lévy, en una entrevista a El País semanal (Hermoso, 202I), habla sobre el papel que acabarían jugando las grandes empresas informáticas y de comunicación, diseñadas como nuevas formas de Estado o Estados-plataforma, siendo que dominan las redes sociales y que cuentan con una infinita capacidad de tratamiento de la información. No lejano a esta reflexión, Facebook ha creado un entorno virtual y sociodigital apto para diferentes actividades culturales y económicas que mantienen como eje la conectividad y la conexión que generan los individuos de sus plataformas, aprovechándose de los vínculos de los propios usuarios.

Entonces, pensar en el ciberespacio como un ambiente social conlleva a pensarlo en diferentes dimensiones, por los efectos que ahí se producen y en particular por los aplicativos que ahí corren, como lo son los medios sociales que recuperan una intangible e inconmensurable información traducida en datos, los datos de cada una de las personas que se suscriben a medios como son las redes sociodigitales.

El uso de Facebook como medio de comunicación permite establecer vínculos digitales con diferentes tipos de usuarios, que a través de esta conectividad virtual ofrece el intercambio simbólico, cultural, económico e incluso emocional. Las personas y empresas en esa fascinación por la conectividad social virtual elevan sus pretensiones y sus ambiciones en el uso, que los llevan a apropiarse de diferentes maneras de la plataforma. Esto puede manifes- 
tarse en diferentes tipos de usuarios, que incluye a los llamados influencers, a los creadores o conversadores digitales que lo son a través de plataformas sociales, utilizadas como canal de comunicación para sus propios intereses.

Es en esta dinámica y éxtasis por la actividad virtual que la comunicación toma relevancia en lo social y tecnológico y la comunicación funciona como un eje de control. Mediación. Así los diferentes usuarios deben aplicar diferentes metodologías y estrategias para comunicarse a través de este medio que como se ha mencionado es un medio que está en una continua transformación. Y como menciona Rovira (2017), las redes sociodigitales cambian la forma en que opera la comunicación esto en tres diferentes dimensiones: la producción, distribución y el consumo.

Debido a este nuevo modelo de comunicación masiva que los medios digitales incorporan, obliga a que las redes sociodigitales deban comprenderse más allá del viejo paradigma del emisor, receptor y mensaje que ocurría de forma unidireccional, para entenderlo desde sus múltiples dimensiones. Como lo es la "autocomunicación de masas" y la autonomía de los sujetos (Castells, 2009).

\section{REFERENCIAS}

Arroyo, I., Calle, S., y Van Wyk, C. (2018). La eficacia en la comunicación de las ONGD. El uso de Facebook en campañas de emergencia. Revista Latina de Comunicación Social, (73), 765-789.

Asencio, A. y Navio, J. (2017). La génesis del Ciberespacio. Universidad Nacional de Educación a Distancia.

Bauman, Z. (2012). Amor líquido: acerca de la fragilidad de los vínculos bumanos. Fondo de Cultura Económica.

Bernete, F. y Cadilla, M. (2019). Usos del ciberespacio para entretenimientos en los ratos libres. Historia y Comunicación Social, 24(2), 579-598. https://doi.org/I0.5209/hics.6630I

Caron, A. y Caronia, L. (2007). Moving cultures: mobile communication in everyday life. McGi11-Queen's University Press.

David, E., Zhitomirsky-Geffet, M., Koppel, M., y Uzan, H. (2016). Utilizing Facebook pages of the political parties to automatically predict the political orientation of Facebook users. Online Information Review, 40(5), 610-623. http://dx.doi.org/I0.IIO8/OIR-09-2015-0308

Del Prado, R. (2018). Ética de la comunicación. Gedisa.

Fuentes, R., Sánchez, E., y Trejo, R. (2011). Quépasa con el estudio de los medios: diálogo con las ciencias sociales en Iberoamérica. Comunicación Social Ediciones y Publicaciones.

Gomes-Franco F. y Colussi, J. (20I6). Uso de Facebook como medio de comunicación alternativo por la "Marcha das Vadias Sampa." Chasqui (I3901079), I3I, 40I-417. 
Herrero, F., Álvarez, A., y López, M. (20II). Revista Latina de Comunicación Social., en la red social Facebook. Revista Latina de Comunicación Social. https://link.gale.com/apps/doc/ A310256802/IFME?u=uan\&sid=bookmark-IFME\&xid=a2d35aO2

Hermoso, B. (202I). Entrevista Pierre Lévy. El País Semanal, 2335, 44-49.

Izquierdo, V., Álvarez, P. y Nuño, A. (20I7). Comunicación y divulgación de contenidos artísticos a través de las Redes Sociales: Facebook y Twitter/Transmitting and disseminating art-related contents through social networks: Facebook and Twitter. Estudios sobre el Mensaje Periodistico, 23(2), II6I. https://link.gale.com/apps/doc/A5306377I2/IFME?u=uan\&sid=bookmark-IFME\&xid=ogaeif46

Introducing Meta: A Social Technology Company. (202I, 28 de octubre). Meta. https://about.fb. com/news/2021/1o/facebook-company-is-now-meta/

Jenkins, H. (2008). Convergence culture: la cultura de la convergencia de los medios de comunicación. Paidós.

Kleiner, D. (20II). Manifesto telecomunista. Ombre corte.

Laudano, C., Corda, M., Planas, J., Kessler, I., y Aracri, A. (2016). Presencia y usos de Facebook en las bibliotecas populares de La Plata, Berisso y Ensenada (Argentina). Información, Cultura y Sociedad, (35), I07.

Lévy, P. (1999). Qué es lo virtual. Paidós.

Lévy, P. (2007). Cibercultura: Informe al Consejo de Europa. Anthropos Editorial Universidad Autónoma Metropolitana.

Losada-Díaz, J. y Capriotti, P. (2015). La comunicación de los museos de arte en Facebook: comparación entre las principales instituciones internacionales y españolas. Palabra-Clave, I8(3), 889-904. https://www.proquest.com/scholarly-journals/la-comunicación-de-los-museos-arte-enfacebook/docview/1726800686/se-2?accountid=4IO2I

Tapia, M. (2016). Los vínculos interpersonales en las redes sociales. Nuevos modos de comunicación en el marco de la Universidad Nacional de San Luis (UNSL), Argentina. Espacio Abierto. Cuaderno Venezolano de Sociología, 25(4), 193-201.

López, G.y Ciuffoli, C.(2012).Facebookes elmensaje: oralidad,escrituray después. La Crujía Ediciones.

Luhmann, N. (2000). La realidad de los medios de masas. Universidad Iberoamericana Anthropos.

Marcos, S., Viounnikoff-Benet, N., y Ripollés, A. (2020). ¿Qué hay en un like? Contenidos políticos en Facebook e Instagram en las elecciones autonómicas valencianas de 20I9. Debats, I34(I), 9III6. http://dx.doi.org/I0.28939/iam.debats.I34-I.6

McLuhan, M. (1996). Comprender los medios de comunicación: las extensiones del ser bumano. Paidós.

Navarro, M., García, I., y Segarra, S. (2020). Utilización de Facebook como canal de comunicación en el sector de la moda: Una comparativa de su vertiente dialógica entre las marcas de moda rápida y de lujo. Palabra-Clave, 23(3), I-3I. http://dx.doi.org/I0.5294/pacla.2020.23.3.5

O'Reilly, T. (2005, 30 de septiembre). What Is Web 2.0. Design Patterns and Business Models for the Next Generation of Software. https://www.oreilly.com/pub/a/web2/archive/what-isweb-20.html 
Pastor, F. y Paniagua, F. (2020). El uso de Facebook como herramienta de comunicación turística en los grandes destinos urbanos internacionales. Doxa Comunicación. Revista Interdisciplinar de Estudios de Comunicación y Ciencias Sociales, (30), 265-28I. https://revistascientificas.uspceu. com/doxacomunicacion/article/view/630

Pilgun, M. y Dzyaloshinsky, I. (2017). Comunicación digital y la realidad social en la comunicación entre los usuarios de las redes sociales rusoparlantes: representación del contenido histórico. $R e$ vista Latina de Comunicación Social, (72), IoIo. https://link.gale.com/apps/doc/A544829465/ $\underline{\text { IFME? }}$ =uan\&sid=bookmark-IFME\&xid=738b604f $\quad \underline{\mathrm{http}} / / / \mathrm{dx}$.doi.org/10.4I85/RLCS2017-I205

Pilgun, M. y Gradoselskaya, G. (2016). Comunicación política en la red social Facebook: segmento rusoparlante I/Political Communication on Facebook: Russian Case. Estudios Sobre El Mensaje Periodístico, 22(2), II6I-II80.

Pisani, F. (2006, 3 de mayo). Arquitectura de participación y negocios. El País. https://elpais.com/ diario/2006/05/04/ciberpais/II46707482_850215.html

Piscitelli, A. (2002). Ciberculturas 2.o: en la era de las máquinas inteligentes. Paidós.

Rodríguez, M., Ruiz, Y., Paino, A., y Jiménez, L. (20I7). El uso de Facebook como herramienta narrativa para articular el nuevo diálogo político. Estudio de caso de la II Asamblea Ciudadana de Podemos, "Vistalegre II“. Revista Latina de Comunicación Social, (72), 849. https://link.gale. com/apps/doc/A544829456/IFME?u=uan\&sid=bookmark-IFME\&xid=35do74ce

Rovira, G. (2017). Activismos en red y multitudes conectadas, comunicación y acción en la era de internet. Icaria.

Segura, G., Paniagua, F., y Fernández, M. (2020). Metodología para evaluar la comunicación universitaria en Facebook y Twitter. Prisma Social, (28), I27-I44. https:/www.proquest.com/ scholarly-journals/metodología-para-evaluar-la-comunicación/docview/235679823I/se-2?accountid $=4 \mathrm{IO} 2 \mathrm{I}$

Thompson, J. (1998). Los media y la modernidad: una teoría de los medios de comunicación. Paidós.

Tubella, I. y Vilaseca, A. (20I3). Sociedad del conocimiento: cómo cambia el mundo ante nuestros ojos. Editorial UOC.

van Dijck, J. (2012). Facebook as a tool for producing sociality and connectivity. Television \& New Media, I3(2), I6o-I76. https://doi.org/Io.II77/I5274764II4I529I

Valera,L., Calvo, D., y López, G. (20I8). Conversaciones políticas en Facebook. Explorando el papel de la homofilia en la argumentación y la interacción comunicativa. Revista Latina de Comunicación Social, (73), 55-73. http://dx.doi.org/10.4185/RLCS-2018-I245

Vromen, A., Loader, D., Xenos, M., y Bailo, F. (2016). Everyday Making through Facebook Engagement: Young Citizens' Political Interactions in Australia, the United Kingdom and the United States. Political Studies, 64(3), 513-533. https://doi.org/I0.II77/00323217156140I2

Vučković, M. y Črnič, T. (2020). Emotionalization and privatization of political communication on facebook: stimulating factors for citizens' interactions? Medijske Studije = Media Studies, II(22), 3-23. http://dx.doi.org/10.2090I/ms.II.22.I 
We are Social \& Hootsuite (2020). Digital 2020: July Global Statshot. https://datareportal.com/ reports/digital-2020-july-global-statshot?rq=covid

We are Social \& Hootsuite. (202I).Digital 202I Global Digital Overview.https://datareportal.com/ reports/digital-202I-july-global-statshot

Zumárraga, M., Silva, L., Trujillo, A. (2020). Determinantes del uso político de Facebook en Ecuador: actitudes, reclutamiento y emociones. América Latina, Hoy, 86, 79-IO2. http://dx.doi. org/IO.I420I/alh.I 8564

Esta obra está bajo Licencia Creative Commons Atribución-NoComercial-SinDerivadas 4.0 Internacional. 